\title{
(DISCRETE) MORSE THEORY ON CONFIGURATION SPACES
}

\author{
Francesca Mori and Mario Salvetti
}

\section{Introduction}

Classical Configuration Spaces in $\mathbb{R}^{d}$ (sometimes written as $F\left(n, \mathbb{R}^{d}\right)$ ) are defined as the set of ordered $n$-tuples of pairwise different points in $\mathbb{R}^{d}$. Taking coordinates in $\left(\mathbb{R}^{d}\right)^{n}=\mathbb{R}^{n d}$

$$
x_{i j}, i=1, \ldots, n, j=1, \ldots, d,
$$

one has

$$
F\left(n, \mathbb{R}^{d}\right)=\mathbb{R}^{n d} \backslash \cup_{i \neq j} H_{i j}^{(d)},
$$

where $H_{i j}^{(d)}$ is the codimension- $d$ subspace

$$
\cap_{k=1, \ldots, d}\left\{x_{i k}=x_{j k}\right\} .
$$

So, the latter subspace is the intersection of $d$ hyperplanes in $\mathbb{R}^{n d}$, each obtained by the hyperplane $H_{i j}=\left\{x \in \mathbb{R}^{n}: x_{i}=x_{j}\right\}$, considered on the $k$-th component in $\left(\mathbb{R}^{n}\right)^{d}=\mathbb{R}^{n d}, k=1, \ldots, d$.

By a Generalized Configuration Space (for brevity, simply a Configuration Space) we mean an analog construction, which starts from any Hyperplane Arrangement $\mathcal{A}$ in $\mathbb{R}^{n}$. For each $d>0$, one has a $d$-complexification $\mathcal{A}^{(d)} \subset \mathbb{R}^{n d}$ of $\mathcal{A}$, which is given by the collection $\left\{H^{(d)}, H \in \mathcal{A}\right\}$ of the $d$-complexified subspaces. The configuration space associated to $\mathcal{A}$ is the complement to the subspace arrangement

$$
\mathcal{M}^{(d)}=\mathcal{M}(\mathcal{A})^{(d)}:=\left(\mathbb{R}^{n}\right)^{d} \backslash \bigcup_{H \in \mathcal{A}} H^{(d)} .
$$

For $d=2$ one has the standard complexification of a real hyperplane arrangement. There is a natural inclusion $\mathcal{M}^{(d)} \hookrightarrow \mathcal{M}^{(d+1)}$ and the limit space is contractible (in case of an arrangement associated to a reflection group $W$, the limit of the orbit space with respect to the action of $W$ gives the classifying space of $W$; see [2]).

The main result of this paper is the explicit construction of a minimal $\mathrm{CW}$-complex for the configuration space $\mathcal{M}(\mathcal{A})^{(d)}$, for all $d \geq 1$. That is, we explicitly produce a $C W$-complex having as many $i$-cells as the $i$-th Betti number $B_{i}$ of $\mathcal{M}(\mathcal{A})^{(d)}, i \geq 0$.

For $d=1$ the result is trivial, since $\mathcal{M}^{(1)}$ is a disjoint union of convex sets (the chambers). The analog result for $d=2$ was found in [12] (see also [16], [3]), after the proof that the complement to the arrangement is a minimal space $([5,14])$. For $d>2$ the configuration spaces are simply-connected, so by general results they have the homotopy type of a minimal $C W$-complex. Nevertheless, having explicit "combinatorial" complexes is useful in order to produce geometric bases for the cohomology. In fact, we give explicit bases for the homology (and cohomology) of $\mathcal{M}^{(d+1)}$ which we

Received by the editors March 30, 2009. 
call (d)-polar bases (see below). As far as we know, there is no other precise description of a geometric $\mathbb{Z}$-basis in the literature, except for some particular arrangements, in spite of the fact that the $\mathbb{Z}$-module structure of the homology is known: it derives from a well known formula in [9] that such homology depends only on the intersection lattice of the $d$-complexification $\mathcal{A}^{(d)}$, and such lattice is the same for all $d \geq 1$.

The main tool which we use is discrete Morse theory for $C W$-complexes, as introduced in $[6,7]$. Starting from the previous explicit construction in [2] of a non-minimal $C W$-complex $\mathbf{S}^{(d)}$, which has the homotopy type of $\mathcal{M}^{(d+1)}$, we construct an explicit combinatorial gradient vector field on $\mathbf{S}^{(d)}$ and we give a precise description of the critical cells. One finds that critical cells live in dimension $i d$, for $i=1, \ldots, n^{\prime}$, where $n^{\prime}$ is the rank of the arrangement $\mathcal{A}\left(n^{\prime} \leq n\right)$.

Notice that the proof of minimality, in case $d>2$, is straightforward from our construction because of the gap between the dimensions of the critical cells.

The above construction depends on a system of polar coordinates in $\mathbb{R}^{n}$, which is generic with respect to the arrangement. Associated to such system is a total ordering $\triangleleft$ (called polar ordering) on the set $\Phi:=\{F\}$ of all the facets of the stratification of $\mathbb{R}^{n}$ induced by the arrangement. Even if the philosophy of the paper is similar to that used for $d=2$ in [12], the extension to the case $d>2$ is not trivial. Let $\prec$ be the standard partial ordering between facets: $F \prec G$ iff $G \subset \operatorname{clos}(F)$. Then the cells of $\mathbf{S}^{(d)}$ correspond to chains $\left(C \prec F_{1} \prec \ldots \prec F_{d}\right)$, where $C$ is a chamber. To construct a gradient field on $\mathbf{S}^{(d)}$, we have to consider on the $i$ th-component of the chains either the polar ordering $\triangleleft_{F_{i+1}}$ which is induced on the arrangement "centered" in the $(i+1)$ th-component, or the opposite $\triangleleft_{F_{i+1}}^{o p}$ of such ordering, according to the parity of $d-i$. Then we use a double induction over $d$ and the dimension of a subarrangement of $\mathcal{A}$.

In section 2 we recall some notations and results from [2], including a description of the complex $\mathbf{S}^{(d)}$.

In section 3 , we recall the main constructions in [12], regarding the case $d=2$.

In section 4 we introduce the degree-d discrete field: we show that it is a gradient field and we characterize the critical cells. We obtain that critical $d k$-cells in $\mathbf{S}^{(d)}$ correspond to chains

$$
\left(C \prec F^{k} \prec \ldots \prec F^{k}\right)
$$

( $k$ is the codimension) if $d$ is odd; to

$$
\left(o p_{F^{k}}(C) \prec F^{k} \prec \ldots \prec F^{k}\right)
$$

if $d$ is even, where $o p_{F^{k}}(C)$ is the chamber opposite to $C$ with respect to $F^{k}$. Here $\left(C \prec F^{k}\right)$ corresponds to a critical cell in $\mathbf{S}^{(1)}$, with respect to polar ordering $\triangleleft$, so it is characterized by

$$
\begin{aligned}
& F \triangleleft G, \quad \forall G \text { such that } \quad F \prec G ; \\
& H \triangleleft F, \quad \forall H \text { such that } \quad C \prec H \prec F .
\end{aligned}
$$

One necessary condition to the minimality of a given space $X$ is: $H^{*}(X ; \mathbb{Z})$ is a free $\mathbb{Z}$-module. For hyperplane complements, this property was known much earlier than the proof of their minimality. It would be interesting to investigate the problem of minimality in general for the case of subspace arrangements. Unlike the case of 
hyperplane arrangements, it is well known that subspace complements with torsion cohomology exist, and they cannot be minimal in the above sense. So, a natural conjecture to consider is: complements to subspace arrangements with torsion-free cohomology are minimal spaces.

\section{Cell decompositions of Configuration Spaces}

In this part we recall some notations and results from [2].

Let $\mathcal{A}=\left\{H_{j}\right\}_{j \in J}$ be a finite arrangement of linear hyperplanes in $M:=\mathbb{R}^{n}$. We introduce a coordinate $x \in M$ and coordinates $\left(x_{1}, \ldots, x_{d}\right), x_{i} \in M$, in $M^{d}, d>0$. Each hyperplane is given by a linear equation $H_{j}=\left\{x \in M: a_{j} \cdot x=0\right\}$, $a_{j} \in M \backslash\{0\}$. For each $d>0$, one has the $d$-complexification $\mathcal{A}^{(d)} \subset M^{d}$ of $\mathcal{A}$, given by the collection of linear codimension- $d$ subspaces

$$
\left(H_{j}\right)^{(d)}:=\left\{\left(x_{1}, \ldots, x_{d}\right): a_{j} \cdot x_{k}=0, k=1, \ldots, d\right\}
$$

(when $d=2$ one has the standard complexification $\mathcal{A}_{\mathbb{C}} \subset \mathbb{C}^{n}$ ).

The generalized configuration space associated to $\mathcal{A}$ is the complement to the subspace arrangement

$$
\mathcal{M}^{(d)}=\mathcal{M}(\mathcal{A})^{(d)}:=M^{d} \backslash \bigcup_{H \in \mathcal{A}} H^{(d)} .
$$

It is convenient to introduce the intersection lattice $\mathcal{L}:=\mathcal{L}(\mathcal{A})$ of $\mathcal{A}$, whose elements are all the subspaces of $M$ of the form

$$
L=H_{j_{1}} \cap \cdots \cap H_{j_{k}}, \quad H_{j_{l}} \in \mathcal{A} .
$$

The partial ordering in $\mathcal{L}$ is given by

$$
L \prec L^{\prime} \text { iff } L^{\prime} \subset L,
$$

so there is a minimum element, corresponding to the empty intersection, which is the whole space $M$, and a maximum $L_{0}:=\bigcap_{H \in A} H$. The $\operatorname{rank} r k(L)$ of a subspace $L \in \mathcal{L}$ is its codimension; the rank of $\mathcal{L}$ is the rank of $L_{0}$, and we also set $r k(\mathcal{A}):=r k(\mathcal{L}(\mathcal{A}))$. The arrangement is called essential when $r k(\mathcal{A})=n=\operatorname{dim}(M)$, i.e. when $L_{0}$ reduces to a single point.

In the present situation we can consider a finer poset $\Phi:=\Phi(\mathcal{A}):=(\{F\}, \prec)$ whose elements are the strata (also called facets) of the stratification induced on $M$ by $\mathcal{A}$, where, as usual:

$$
F \prec F^{\prime} \text { iff } F^{\prime} \subset \operatorname{cl}(F) .
$$

The atoms (chambers) of $\Phi(\mathcal{A})$ are the connected components of $\mathcal{M}^{(1)}$.

We have a map $\Phi \rightarrow \mathcal{L}$ which associates to a facet $F$ its support $|F|$, which is by definition the subspace generated by $F$ (in a different language, this is the standard map between an oriented matroid and its underlying matroid). We define the rank function on $\Phi$ via this map:

$$
r k(F):=r k(|F|)=\operatorname{codim}(F) .
$$

Given $L \in \mathcal{L}(\mathcal{A})$, we will use the arrangements $\mathcal{A}_{L}:=\{H \in \mathcal{A}: H \prec L\}$, $\mathcal{A}^{L}:=\{L \cap H: H \in \mathcal{A}, H \nprec L\}$. The former is an arrangement in $M$ of rank equal to $r k(L)$, the latter is an arrangement inside $L$ itself (of rank $r k(\mathcal{A})-r k(L)$ ). Let $\Phi_{L}:=\Phi\left(\mathcal{A}_{L}\right), \Phi^{L}:=\Phi\left(\mathcal{A}^{L}\right)$ be the induced stratifications of $M, L$ respectively. 
There is a map $p r_{L}: \Phi \rightarrow \Phi_{L}$, taking $F^{\prime}$ into the unique stratum containing it, and a map $j^{L}: \Phi^{L} \rightarrow \Phi$ just given by the inclusion.

Fixing a facet $F$, set also $\Phi_{F}=\left\{F^{\prime} \in \Phi: F^{\prime} \prec F\right\}$. It is easy to see that the restriction $\varphi_{F}:=p r_{|F|} \mid \Phi_{F}: \Phi_{F} \rightarrow \Phi_{|F|}$ is a dimension-preserving bijection of posets.

Let now $\Phi^{d}$ be the product of $d$ copies of $\Phi, d \geq 0$, and let

$$
\Phi^{(d)}=\left\{\left(F_{1}, \ldots, F_{d}\right) \in \Phi^{d}: F_{1} \prec \ldots \prec F_{d}\right\}
$$

be the set of $d$-chains in $\Phi$ (repetitions in the chain are allowed). Then $\Phi^{(d)}$ corresponds to a stratification of the space $M^{d}$ as follows (see [2]): to each $\mathcal{F}=$ $\left(F_{1}, \ldots, F_{d}\right)$ in $\Phi^{(d)}$ it corresponds the stratum $\hat{\mathcal{F}}$ in $M^{d}$ given by

$$
\hat{\mathcal{F}}:=\left\{\left(x_{1}, \ldots, x_{d}\right) \in M^{d}: x_{1} \in F_{d}, x_{k} \in \varphi_{F_{d-k+2}}\left(F_{d-k+1}\right), k=2, \ldots, d\right\} .
$$

One has

Proposition 2.1. (i) Each $\hat{\mathcal{F}}$ is homeomorphic to an open cell

(ii) $\bigcup_{\mathcal{F} \in \Phi^{(d)}} \quad \hat{\mathcal{F}}=M^{d}$

(iii) $\hat{\mathcal{F}} \cap \hat{\mathcal{G}}=\emptyset$ if $\mathcal{F} \neq \mathcal{G}$

(iv) $\operatorname{cl}(\hat{\mathcal{F}}) \cap \hat{\mathcal{G}} \neq \emptyset$ iff $\operatorname{cl}(\hat{\mathcal{F}}) \supset \hat{\mathcal{G}}$.

(v) $\mathcal{M}^{(d)}=\bigcup_{\left\{\mathcal{F} \in \Phi^{(d)}\right.}: F_{1}$ is a chamber of $\left.\Phi\right\} \hat{\mathcal{F}}$

For $\mathcal{F}=\left(F_{1}, \ldots, F_{d}\right)$, one has

$$
\operatorname{codim}(\mathcal{F}):=\operatorname{codim}(\hat{\mathcal{F}})=\sum_{i=1}^{d} \operatorname{codim}\left(F_{i}\right) .
$$

The partial ordering on $\Phi^{(d)}$ is given by

$$
\mathcal{F} \prec \mathcal{G} \quad \text { iff } \quad \hat{\mathcal{G}} \subset \operatorname{cl}(\hat{\mathcal{F}}) .
$$

This has the following characterization.

Lemma 2.2. For $\mathcal{F}=\left(F_{1}, \ldots, F_{d}\right), \mathcal{G}=\left(G_{1}, \ldots, G_{d}\right) \in \Phi^{(d)}$ one has

$$
\mathcal{F} \prec \mathcal{G} \quad \text { iff } F_{d} \prec G_{d} \quad \text { and } p r_{\left|F_{i+1}\right|}\left(F_{i}\right) \prec p r_{\left|F_{i+1}\right|}\left(G_{i}\right)
$$

in the stratification $\Phi_{\left|F_{i+1}\right|}, i=d-1, \ldots, 1$.

Part (v) of proposition 2.1 gives us the poset corresponding to the induced stratification of the generalized configuration space $\mathcal{M}^{(d)}$ which is

$$
\Phi_{0}^{(d)}:=\left\{\mathcal{F}=\left(F_{1}, \ldots, F_{d}\right) \in \Phi^{(d)}: r k\left(F_{1}\right)=0\right\}
$$

while the union $\bigcup_{H \in A} H^{(d)}$ of the $d$-complexified subspaces correspond to the poset

$$
\Phi_{+}^{(d)}:=\left\{\mathcal{F}=\left(F_{1}, \ldots, F_{d}\right) \in \Phi^{(d)}: \operatorname{rk}\left(F_{1}\right)>0\right\} .
$$

Proposition 2.3. The set

$$
\mathbf{Q}^{(d)}:=\bigcup_{\mathcal{F} \in \Phi^{(d)}} e(\mathcal{F}),
$$

where $e(\mathcal{F})$ is the dual cell to the stratum $\mathcal{F}$, is a cellular $n^{\prime} d$-ball in $M^{d}$ (a regular cell complex) dual to the stratification, where $n^{\prime}:=\operatorname{rk}\left(L_{0}\right)$. 
Remark. It follows from lemma 2.2 that if the first element $F_{1}$ of $\mathcal{F}$ is a chamber, then also the first element of any $\mathcal{G} \prec \mathcal{F}$ is a chamber.

Definition 2.4. We denote by $\mathbf{S}^{(d)}$ the subcomplex of $\mathbf{Q}^{(d+1)}$ whose cells correspond to $\Phi_{0}^{(d+1)}$ :

$$
\mathbf{S}^{(d)}:=\cup_{\mathcal{F} \in \Phi_{0}^{(d+1)}} e(\mathcal{F})
$$

(case $d=1$ was introduced in [11]; see also [1], [13]).

There is a natural inclusion

$$
j_{d}: \mathbf{S}^{(d)} \rightarrow \mathbf{S}^{(d+1)}, \quad j_{d}\left(e\left(C, F_{1}, \cdots, F_{d}\right)\right)=e\left(C, C, F_{1}, \cdots, F_{d}\right) .
$$

For $\mathcal{F}=\left(C, F_{1}, F_{2}, \cdots\right)$ we write $e\left(C, \mathcal{F}^{\prime}\right):=e(\mathcal{F})$, with $\mathcal{F}^{\prime}=\left(F_{1}, F_{2}, \cdots\right)$. In general, given a chamber $C$ and a facet $F$ in $\Phi$ we will use the notation

$$
C . F:=\varphi_{F^{-1}}\left(p r_{|F|}(C)\right)
$$

which is a uniquely defined chamber containing $F$ in its boundary.

One has:

Theorem 1. (i) $\mathbf{S}^{(d)}$ is a deformation retract of $\mathcal{M}^{(d+1)}$.

(ii)

$$
\partial\left(e\left(C, \mathcal{F}^{\prime}\right)\right)=\bigcup_{\mathcal{F}^{\prime \prime} \prec \mathcal{F}^{\prime}, \operatorname{codim}\left(\mathcal{F}^{\prime \prime}\right)=\operatorname{codim}\left(\mathcal{F}^{\prime}\right)-1} e\left(C \cdot F_{1}, \mathcal{F}^{\prime \prime}\right)
$$

$\left(\mathcal{F}^{\prime \prime}=\left(F_{1}, \ldots\right)\right)$.

(iii) $\operatorname{dim} e\left(C, \mathcal{F}^{\prime}\right)=\operatorname{dim} e\left(\mathcal{F}^{\prime}\right)=\operatorname{codim}_{M^{d}}\left(\mathcal{F}^{\prime}\right)$. In particular $\operatorname{dim}\left(\mathbf{S}^{(d)}\right)=$ $\operatorname{dim}\left(\mathbf{Q}^{(d)}\right)=n^{\prime} d$.

\section{Discrete Morse theory on Hyperplane Arrangements}

We refer here to the main definitions and results from [6], [7], where Morse theory from a combinatorial viewpoint was first developed (see also [10], where an approach to Discrete Morse Theory is developed, where discrete gradient vector fields are replaced by acyclic matchings over a poset).

In the remaining of section 3 we need a brief summary (skipping some details) of some of the results in [12], where we applied the theory to Hyperplane Arrangements. We set in this section $\mathbf{S}:=\mathbf{S}^{(1)}$, the case of standard complexification. A $k$-cell of $\mathbf{S}$ is written as $e(\mathcal{F}), \mathcal{F}=(C, F)$, with $\operatorname{codim}(F)=k$. We write also $e(\mathcal{F})=e(C, F)$. The boundary condition given in lemma 2.2 specializes here to: $e(D, G)$ is in the boundary of $e(C, F)$ iff

i) $G \prec F$

ii) the chambers $C$ and $D$ are contained in the same chamber of $\mathcal{A}_{G}$, that is $D=C . G$ in the notations of part 2 .

The following constructions are based on a generic system of polar coordinates in $M$, which is associated to a generic flag of subspaces

$$
V_{i}=<\mathbf{e}_{1}, \ldots, \mathbf{e}_{i}>, \quad i=0, \ldots, n\left(\operatorname{dim}\left(V_{i}\right)=i\right) .
$$


Consider the pencil of $(n-1)$-dimensional subspaces of $M, V_{n-1}(\theta), \theta \in \mathbb{R}$, with base $V_{n-2}$ : so $V_{n-1}(0)=V_{n-1}$ and $\theta$ grows according to some positive orientation of $V / V_{n-2}$. By recurrence, let $V_{i}\left(\theta, \theta_{i+1}, \ldots, \theta_{n-1}\right), \theta \in \mathbb{R}$, be the pencil of $i$ dimensional subspaces in $V_{i+1}\left(\theta_{i+1}, \ldots, \theta_{n-1}\right)$, with base $V_{i-1}, i=1, \ldots, n-2$. Let also $V_{0}\left(\theta, \theta_{1}, \ldots, \theta_{n-1}\right)$ be the point with distance $\theta$ from the origin $V_{0}$ inside the line $V_{1}\left(\theta_{1}, \ldots, \theta_{n-1}\right)$.

Each point $P \neq V_{0}$ is written uniquely as $P=V_{0}\left(\theta_{0}, \theta_{1}, \ldots, \theta_{n-1}\right)$, so we associate to $P$ the polar coordinates $\left(\theta_{0}, \ldots, \theta_{n-1}\right)$.

Definition 3.1. We say that the above flag is generic with respect to the arrangement $\mathcal{A}$ if it satisfies the following conditions:

i) the origin $V_{0}$ is contained in a chamber $C_{0}$ of $\mathcal{A}$;

ii) there exists a $\delta>0, \delta<<\pi / 2$, such that the set of bounded facets of $\mathcal{A}$ is contained into $B(\delta):=\left\{P \in M: 0<\theta_{i}(P)<\delta, i=0, \ldots, n-1\right\}$;

iii) subspaces $V_{i}(\bar{\theta})=V_{i}\left(\bar{\theta}_{i}, \ldots, \bar{\theta}_{n-1}\right)$ which intersect $B(\delta)$ are generic with respect to $\mathcal{A}$, in the sense that, for each codim-k subspace $L \in L(\mathcal{A})$,

$$
\operatorname{dim}\left(\left|V_{i}(\bar{\theta})\right| \cap L\right)=i-k .
$$

One proves that a generic system of polar coordinates always exists.

Fix a system of polar coordinates associated to a generic flag.

We consider the subspace $V_{i}(\bar{\theta}), \bar{\theta}=\left(\bar{\theta}_{i}, \ldots, \bar{\theta}_{n-1}\right), \theta_{j} \in[0, \delta], j=i, \ldots, n-1$. The arrangement $\mathcal{A}$ induces a stratification $\Phi(\bar{\theta})$ in $V_{i}(\bar{\theta})$ : given a codimension- $k$ facet $F \in \Phi$, let us denote by

$$
F(\bar{\theta}):=F \cap V_{i}(\bar{\theta}) .
$$

By genericity conditions, if $i \geq k$ then $F(\bar{\theta})$ is either empty or it is a codimension $k+n-i$ facet contained in $V_{i}(\bar{\theta})$.

For each such $\bar{\theta}$ we want to give a total ordering on $\Phi(\bar{\theta})$.

Definition 3.2. Given any facet $F(\bar{\theta})$ let us denote by

$$
P_{F(\bar{\theta})} \in \operatorname{clos}(F(\bar{\theta}))
$$

the "minimum" vertex of $\operatorname{clos}(F(\bar{\theta})) \cap \mathbb{R}_{\geq 0}^{n}$ : this is the 0-dimensional facet which has minimum polar coordinates, with respect to the anti-lexicographic ordering of the coordinates (so, starting from $\theta_{n-1}$ and going back).

Remark that the minimum vertex is well defined by genericity conditions.

Definition 3.3. We define the polar ordering by recurrence on the dimension as follows: given $F, G \in \Phi$, and given $\bar{\theta}=\left(\bar{\theta}_{i}, \ldots, \bar{\theta}_{n-1}\right), 0 \leq i \leq n, \bar{\theta}_{j} \in[0, \delta]$ for $j \in i, . ., n-1,(\bar{\theta}=\emptyset$ for $i=n)$ such that $F(\bar{\theta}), G(\bar{\theta}) \neq \emptyset$, we set

$$
F(\bar{\theta}) \triangleleft G(\bar{\theta})
$$

iff one of the following cases occurs:

i) $P_{F(\bar{\theta})} \neq P_{G(\bar{\theta})}$ and the coordinates of the former point are lower (in the antilexicographical ordering) than the coordinates of the latter point.

ii) $P_{F(\bar{\theta})}=P_{G(\bar{\theta})}$. Then either

iia) $\operatorname{dim}(F(\bar{\theta}))=0 \quad\left(\right.$ so $\left.P_{F(\bar{\theta})}=F(\bar{\theta})\right)$ and $F(\bar{\theta}) \neq G(\bar{\theta}) \quad(\operatorname{sodim}(G(\bar{\theta}))>0)$ 
or

iib) $\operatorname{dim}(F(\bar{\theta}))>0, \operatorname{dim}(G(\bar{\theta}))>0$.

Let $i_{0}:=\max \left\{j \mid \theta_{j}\left(P_{F(\bar{\theta})}\right) \neq 0\right\}+1$ and let $i_{1}:=\min \left\{i_{0}, i\right\}$. If the coordinates of the minimal point are $P \equiv\left(\bar{\theta}_{0}, \ldots, \bar{\theta}_{n-1}\right)$ then $\forall \epsilon, 0<\epsilon<<\delta$, it must happen

$$
F\left(\bar{\theta}_{i_{1}-1}+\epsilon, \bar{\theta}_{i_{1}}, \ldots\right) \triangleleft G\left(\bar{\theta}_{i_{1}-1}+\epsilon, \bar{\theta}_{i_{1}}, \ldots\right) .
$$

The idea in (iib) is to intersect $F$ and $G$ with a lower dimensional subspace contained in $V_{i}(\bar{\theta})$, very close to the minimum point (see also [4] for a purely combinatorial version of polar ordering).

One shows that polar ordering $\triangleleft$ is a total ordering on the facets of $V_{i}(\bar{\theta})$, for any given $\bar{\theta}=\left(\bar{\theta}_{i}, \ldots, \bar{\theta}_{n-1}\right)$. In particular (taking $\bar{\theta}=\emptyset$ ) it gives a total ordering on $\Phi$.

We consider now the regular CW-complex $\mathbf{S}=\mathbf{S}^{(1)}$ and we define (see [6], [7]) a combinatorial gradient vector field $\Gamma$ over $\mathbf{S}$. One can describe $\Gamma$ as a collection of pairs of cells

$$
\Gamma \subset\{(e, f) \in \mathbf{S} \times \mathbf{S} \mid \operatorname{dim}(f)=\operatorname{dim}(e)+1, e \in \partial(f)\}
$$

so that $\Gamma$ decomposes into its dimension-p components

$$
\Gamma=\bigsqcup_{p=1}^{n} \Gamma^{p}, \quad \Gamma^{p} \subset \mathbf{S}_{p-1} \times \mathbf{S}_{p}
$$

$\left(\mathbf{S}_{p}\right.$ being the $p$-skeleton of $\left.\mathbf{S}\right)$.

We give the following recursive definition:

Definition 3.4 (Polar Gradient). We define a combinatorial gradient field $\Gamma$ over $\mathbf{S}$ in the following way:

the $(j+1)$-th component $\Gamma^{j+1}$ of $\Gamma, j=0, \ldots, n-1$, is given by all pairs

$$
\left(e\left(C, F^{j}\right), e\left(C, F^{j+1}\right)\right), \quad F^{j} \prec F^{j+1}
$$

(same chamber $C$ ) such that

(1) $F^{j+1} \triangleleft F^{j}$

(2) $\forall F^{j-1} \prec F^{j}$ such that $C \prec F^{j-1}$ the pair

$$
\left(e\left(C \prec F^{j-1}\right), e\left(C \prec F^{j}\right)\right) \notin \Gamma^{j}
$$

Theorem 2. One has:

(1) $\Gamma$ is a combinatorial vector field on $\mathbf{S}$ which is the gradient of a discrete Morse function.

(2) The pair

$$
\left(e\left(C \prec F^{j}\right), e\left(C \prec F^{j+1}\right)\right), \quad F^{j} \prec F^{j+1}
$$

belongs to $\Gamma$ iff the following conditions hold:

(a) $F^{j+1} \triangleleft F^{j}$

(b) $\forall F^{j-1}$ such that $C \prec F^{j-1} \prec F^{j}$, one has $F^{j-1} \triangleleft F^{j}$. 
(3) Given $F^{j} \in \Phi$, there exists a chamber $C$ such that the cell $e\left(C \prec F^{j}\right)$ is the second factor of a pair in $\Gamma$ iff there exists $F^{j-1} \prec F^{j}$ with $F^{j} \triangleleft F^{j-1}$. More precisely, for each chamber $C$ such that there exists $F^{j-1}$ with

$$
C \prec F^{j-1} \prec F^{j}, \quad F^{j} \triangleleft F^{j-1}
$$

the pair $\left(e\left(C \prec \bar{F}^{j-1}\right), e\left(C \prec F^{j}\right)\right) \in \Gamma$, where $\bar{F}^{j-1}$ is the maximum $(j-1)$-facet (with respect to polar ordering) satisfying conditions $\left(^{*}\right)$.

(4) The set of $k$-dimensional critical cells is given by

$$
\begin{aligned}
& \operatorname{Sing}_{k}(\mathbf{S})= \\
& \quad=\left\{e\left(C \prec F^{k}\right): F^{k} \cap V_{k} \neq \emptyset, F^{j} \triangleleft F^{k}, \forall C \prec F^{j} \supsetneqq F^{k}\right\} .
\end{aligned}
$$

Equivalently, $F^{k} \cap V_{k}$ is the maximum (in polar ordering) among all facets of $C \cap V_{k}$.

In section 4 we will need some more specific results which were used in the proof of part (1) of theorem 2.

Take a $\Gamma$-path in $\mathbf{S}$ (according to [7])

$$
e\left(C_{1}, F_{1}^{k}\right), e\left(C_{1}, F_{1}^{k+1}\right), \ldots, e\left(C_{m}, F_{m}^{k}\right), e\left(C_{m}, F_{m}^{k+1}\right), e\left(C_{m+1}, F_{m+1}^{k}\right)
$$

Here the pair $\left(e\left(C_{i}, F_{i}^{k}\right), e\left(C_{i}, F_{i}^{k+1}\right)\right)$ is an element of $\Gamma$, and $e\left(C_{i}, F_{i}^{k}\right)$ is in the boundary of $e\left(C_{i-1}, F_{i-1}^{k+1}\right)$.

According to a standard result in Discrete Morse Theory ([6]) we have to show that, if the path (2) is closed, (i.e. if $e\left(C_{m+1}, F_{m+1}^{k}\right)$ equals to $\left.e\left(C_{1}, F_{1}^{k}\right)\right)$, then it is trivial, i.e. $F_{i}^{k}=F_{i+1}^{k}, F_{i}^{k+1}=F_{i+1}^{k+1}$, and $C_{i}=C_{i+1}(i=1, \ldots, m-1)$.

The proof directly follows from the following two claims.

Claim 1. Given a triple of consecutive cells in (2) of the form:

$$
e\left(C_{i}, F_{i}^{k+1}\right), e\left(C_{i+1}, F_{i+1}^{k}\right), e\left(C_{i+1}, F_{i+1}^{k+1}\right) .
$$

we have that $F_{i+1}^{k+1} \unlhd F_{i}^{k+1}$.

Claim 2. Given a quadruple of consecutive cells in (2) of the form:

$$
e\left(C_{i}, F_{i}^{k}\right), e\left(C_{i}, F^{k+1}\right), e\left(C_{i+1}, F_{i+1}^{k}\right), e\left(C_{i+1}, F^{k+1}\right) .
$$

we have $F_{i}^{k} \unlhd F_{i+1}^{k}$.

Remark 3.5. 1) Once a polar ordering is assigned, the set of singular cells is described only in terms of it by

$\operatorname{Sing}_{k}(\mathbf{S}):=\left\{e\left(C \prec F^{k}\right):\right.$

a) $F^{k} \triangleleft F^{k+1}, \quad \forall \quad F^{k+1} \quad$ s.t. $\quad F^{k} \prec F^{k+1}$

b) $\quad F^{\prime} \triangleleft F^{k}, \quad \forall \quad F^{\prime} \quad$ s.t. $\left.C \prec F^{\prime} \prec F^{k}\right\}$

2) The construction of theorem 2 gives an explicit additive basis for the homology and for the cohomology in terms of the singular cells in $\mathbf{S}$. We can call it a polar basis (relative to a given system of generic polar coordinates).

3) The minimality of the associated Morse complex is obtained by the one-to-one correspondence between singular cells and the set of all the chambers of $\Phi$, and the well-known formula $\sum b_{i}=\mid\{$ chambers $\} \mid$ (see [15]). 


\section{Discrete Morse theory on Configuration Spaces}

We now consider general configuration spaces as in part 2, generalizing the theory of part 3. We fix a polar ordering $\triangleleft$ induced by a generic system of polar coordinates $V_{0}, V_{1}, \ldots, V_{n}$ as in part 3 .

Lemma 4.1. Let $L \in \mathcal{L}(\mathcal{A})$ be a codimension- $k$ subspace. Then the system $V_{0}, \ldots, V_{k}$ gives a generic system of polar coordinates for the arrangement $\mathcal{A}_{L} \cap V_{k}:=\{H \cap$ $\left.V_{k} \mid H \in \mathcal{A}\right\}$ in $V_{k}$, so it induces a polar ordering $\triangleleft_{L}$ on $\Phi_{L}$. The system $V_{k} \cap L, \ldots, V_{n} \cap$ $L$ gives a generic system of polar coordinates for the arrangement $\mathcal{A}^{L}$ on $L$, inducing a polar ordering $\triangleleft^{L}$ on $\Phi^{L}$.

One has that $\triangleleft^{L}$ coincides with the restriction $\triangleleft_{\Phi^{L}}$ of the polar ordering $\triangleleft$ to $\Phi^{L} \subset \Phi$.

Proof. In the case of $\Phi_{L}$, there are no bounded facets, except for the point $L \cap V_{k}$, and the genericity condition reduces to transversality, which is included in the genericity condition for the given system $V_{0}, \ldots, V_{n}$, taking into account that $\mathcal{L}\left(\mathcal{A}_{L}\right) \subset \mathcal{L}(\mathcal{A})$.

For $\Phi^{L}$, just remark that by the genericity of the given system, $V_{i} \cap L \neq \emptyset$ only for $i \geq k$, so by the genericity of the original system one gets the genericity of the restricted one $V_{k} \cap L, \ldots, V_{n} \cap L$. Now notice that if $\left(\theta_{0}^{\prime}, \ldots, \theta_{n-k}^{\prime}\right)$ are the polar coordinates of a point $P$ in $L$ with respect to the system $V_{k} \cap L, \ldots, V_{n} \cap L$ and $\left(\theta_{0}, \ldots, \theta_{n}\right)$ are the polar coordinates of $P$ with respect to $V_{0}, \ldots, V_{n}$, then there exist smooth functions:

$$
\alpha_{j}:([0, \epsilon))^{n-j+1} \rightarrow \mathbb{R}_{\geq 0}:\left(\theta_{j}, \ldots, \theta_{n}\right) \mapsto \theta_{j}^{\prime}, j=k, \ldots, n,
$$

with $\epsilon>\delta(\delta$ as in 3.1) such that:

$$
\theta_{i}^{\prime}=\alpha_{i+k}\left(\theta_{i+k}, \ldots, \theta_{n}\right), \quad i=0, \ldots, n-k
$$

and the function:

$$
\theta \longmapsto \alpha_{i+k}\left(\theta, \theta_{i+k+1}, \ldots, \theta_{n}\right)
$$

is strictly increasing in $[0, \epsilon)(i=0, \ldots, n-k)$. This shows the last assertion of the lemma, by definition of polar ordering (see part 3 ).

Definition 4.2. Given $G \in \Phi$, we set $\triangleleft_{G}:=\triangleleft_{|G|}$.

Definition 4.3. Given $G \in \Phi$, and being $\Phi_{G}$ as in part 2, we define an involution:

$$
\begin{aligned}
o p_{G}: \Phi_{G} & \rightarrow \Phi_{G} \\
F & \mapsto o p_{G}(F)
\end{aligned}
$$

where $\operatorname{op}_{G}(F)$ is the unique facet which is symmetric to $F$ with respect to $\operatorname{supp}(G)$. In other terms (using the maps $\varphi_{G}, p r_{|G|}$ defined in section 2):

$$
o_{G}(F):=\varphi_{G}^{-1}\left(-\left(p r_{|G|}(F)\right)\right) .
$$

Here we notice that, for a central arrangement, every facet $F$ has a unique opposite $-F$ with respect to the center. 
Definition 4.4. Define the opposite polar ordering $\triangleleft_{G}^{o p}$ in $\Phi_{G}$ as:

$$
F \triangleleft_{G}^{o p} F^{\prime} \Leftrightarrow o p_{G}(F) \triangleleft_{G} o p_{G}\left(F^{\prime}\right)
$$

$F, F^{\prime} \in \Phi_{G}$. One can show that $\triangleleft_{G}^{o p}$ is the polar ordering with respect to a system $V_{0}^{\prime}, \cdots, V_{k}^{\prime}, k=\operatorname{codim}(G)$, where $V_{i}^{\prime}$ is the symmetric of $V_{i}$ with respect to the point $V_{k} \cap|G|$.

Definition 4.5. For all arrangements $\mathcal{A}$, all polar orderings $\triangleleft$ on $\mathcal{A}$, and all $d \geq 1$, we define the degree-d discrete field

$$
\Gamma_{(d)}:=\Gamma_{(d)}(\mathcal{A}, \triangleleft)
$$

on the complex $\mathbf{S}^{(d)}(\mathcal{A})$. Assume by recurrence that $\Gamma_{\left(d^{\prime}\right)}\left(\mathcal{A}^{L}\right)$ has been defined for $d^{\prime}<d$, for any $\mathcal{A}^{L} \subset \mathcal{A}, L \in \mathcal{L}(\mathcal{A})$, for the induced polar ordering $\triangleleft^{L}$ (see lemma 4.1). Then the $k$-dimensional part $\Gamma_{(d)}^{k}(\mathcal{A})$ is given by the set of pairs of cells in $\mathbf{S}^{(d)}(\mathcal{A})$

$$
\left(e(\mathcal{F}), e\left(\mathcal{F}^{\prime}\right)\right)
$$

where $\operatorname{dim}(e(\mathcal{F}))=k-1, \operatorname{dim}\left(e\left(\mathcal{F}^{\prime}\right)\right)=k, \mathcal{F} \prec \mathcal{F}^{\prime}\left(\operatorname{so} e(\mathcal{F}) \subset \partial\left(e\left(\mathcal{F}^{\prime}\right)\right)\right)$, and the two flags differ only in a single position:

$$
\begin{aligned}
\mathcal{F} & =\left(C, F_{1}, \ldots, F_{i-1}, F_{i}^{j}, F_{i+1}, \ldots, F_{d}\right), \\
\mathcal{F}^{\prime} & =\left(C, F_{1}, \ldots, F_{i-1}, F_{i}^{j+1}, F_{i+1}, \ldots, F_{d}\right)
\end{aligned}
$$

with $F_{i}^{j} \prec F_{i}^{j+1}(j, j+1$ denote codimensions). Moreover, such pairs satisfy the following conditions (5),(6):

for $i<d, e\left(F_{i}^{j}, F_{i+1}, \ldots, F_{d}\right)$ is a critical cell in the complex $\mathbf{S}^{(d-i)}\left(\mathcal{A}^{L}\right)$, endowed with the discrete $(d-i)$ - vector field $\Gamma_{(d-i)}\left(\mathcal{A}^{L}\right)$, with $L:=\left|F_{i}^{j}\right|$ (i.e., $e\left(F_{i}^{j}, F_{i+1}, \ldots, F_{d}\right) \notin \Gamma_{(d-i)}\left(\mathcal{A}^{L}\right)$, $\operatorname{see}([6,7]))$

Set $l=d-i$; then:

$$
\begin{aligned}
& \text { for even } l \\
& \quad F_{i}^{j+1} \triangleleft_{F_{i+1}} F_{i}^{j} \quad \text { and } \quad F_{i}^{j}=\max _{\triangleleft F_{i+1}}\left\{F \mid F_{i-1} \prec F \prec F_{i}^{j}\right\} ;
\end{aligned}
$$

for odd $l$

$$
F_{i}^{j+1} \triangleleft_{F_{i+1}}^{o p} F_{i}^{j} \quad \text { and } \quad F_{i}^{j}=\max _{\triangleleft_{F_{i+1}}^{o p}}\left\{F \mid F_{i-1} \prec F \prec F_{i}^{j}\right\} \text {. }
$$

For $i=d$ there is no $F_{i+1}$ in the first condition of (6), which is to be considered in this case as defined by using the given polar ordering $\triangleleft$.

We have $\Gamma_{(d)}=\oplus_{k=1}^{n^{\prime} d} \Gamma_{(d)}^{k}, n^{\prime}=\operatorname{rk}(\mathcal{A})$.

Definition 4.6. Let $L \in \mathcal{L}(\mathcal{A})$ be a codimension $k$ subspace.

Set $\Gamma_{(d)}^{L}$ as the degree-d discrete field of the arrangement $\mathcal{A}^{L}$ with respect to the polar ordering $\triangleleft^{L}=\triangleleft_{\left.\right|_{L}}$.

Set $\Gamma_{L,(d)}$ as the degree-d discrete field of the arrangement $\mathcal{A}_{L} \cap V^{k}$ with respect to the polar ordering $\triangleleft_{L}$ (see lemma (4.1)). 
The following main theorem describes the minimal complex in terms of the field $\Gamma_{(d)}$, exhibiting its critical cells.

Theorem 3. One has:

(1) $\Gamma_{(d)}$ is a discrete vector field;

(2) $\Gamma_{(d)}$ is the gradient field of a discrete Morse function;

(3) the critical cells of $\Gamma_{(d)}$ are the following ones, depending on the parity of $d$ :

$$
e\left(C, F^{k}, \ldots, F^{k}\right)
$$

with $e\left(C, F^{k}\right) \in \mathbf{S}^{(1)}$ critical cell for $\left(\Gamma_{(1)}, \triangleleft\right)$, if $d$ is odd;

$$
e\left(o p_{F^{k}}(C), F^{k}, \ldots, F^{k}\right)
$$

with $e\left(C, F^{k}\right) \in \mathbf{S}^{(1)}$ critical cell for $\left(\Gamma_{(1)}, \triangleleft\right)$, if $d$ is even.

As an immediate consequence of theorem 3 we have

Theorem 4. (1) The configuration space $\mathcal{M}^{(d)}(\mathcal{A})$ is a minimal space $(d \geq 1)$.

(2) The cohomology of $\mathbf{S}^{(d)}$ (or of $\mathcal{M}^{(d+1)}$ ), $d \geq 1$, is concentrated in dimension

$$
i d, i=0 \ldots n \text {. }
$$

The Betti numbers are given by

$$
B_{i d}\left(\mathbf{S}^{(d)}\right)=B_{i}\left(\mathbf{S}^{(1)}\right) .
$$

Proof of theorem 4. Case $d=1$ is considered in section 3. For $d>1$ minimality follows immediately from the gap between the dimensions of the critical cells.

Remark. For $d>1$ the configuration space is simply connected, therefore its minimality follows by general means. For $d=1$ there is also a non trivial Morse complex for local cohomology (see [12],[8]).

Notation 4.7. Given $L \in \mathcal{L}(\mathcal{A})$, with $\operatorname{codim}(L)=k$, notice that the cells of $\mathbf{S}^{(d-1)}\left(\mathcal{A}^{L}\right)$ have the form $e(\mathcal{F})$, where $\mathcal{F}$ is a flag whose first element is some facet $F^{k}$ with $\left|F^{k}\right|=L$ (in fact, $F^{k}$ is a chamber in the arrangement $\mathcal{A}^{L}$ ).

Let

$$
\lambda: \mathbf{S}^{(d-1)}\left(\mathcal{A}^{L}\right) \rightarrow \mathbf{S}^{(d)}(\mathcal{A})
$$

be the correspondence defined by

$$
\lambda\left(e\left(F^{k}, F_{2}, \ldots, F_{d-1}\right)\right):=\left\{e\left(C, F^{k}, F_{2}, \ldots, F_{d-1}\right) \mid C \prec F^{k}\right\} \subset \mathbf{S}^{(d)}(\mathcal{A}) .
$$

When $\Gamma^{\prime}$ is a discrete field over $\mathbf{S}^{(d-1)}\left(\mathcal{A}^{L}\right)$, we have an induced field $\lambda_{*}\left(\Gamma^{\prime}\right)$ over $\mathbf{S}^{(d)}(\mathcal{A})$ : for each pair

$$
\left(e\left(F^{k}, F_{2}, \ldots, F_{d-1}\right), e\left(F^{k}, F_{2}^{\prime}, \ldots, F_{d-1}^{\prime}\right)\right) \in \Gamma^{\prime},
$$

take all pairs of the shape

$$
\left(e\left(C, F^{k}, F_{2}, \ldots, F_{d-1}\right), e\left(C, F^{k}, F_{2}^{\prime}, \ldots, F_{d-1}^{\prime}\right)\right) \text {, for all chambers } C \prec F^{k} \text {. }
$$

In particular, when $L$ is the whole space $M, \lambda$ gives a map which to a discrete field on $\mathbf{S}^{(d-1)}(\mathcal{A})$ associates a discrete field on $\mathbf{S}^{(d)}(\mathcal{A})$ (in this case $k=0$ so one can add just one chamber $\left.C=F^{0}\right)$. Here $\lambda=j_{d-1}: \mathbf{S}^{(d-1)} \rightarrow \mathbf{S}^{(d)}$, defined at the end of part 2. 
Proof of theorem 3. We proceed by double induction on the degree $d$ and the rank of the arrangement $\mathcal{A}$.

The case $d=1$, any rank, is exactly given by theorem 2 , part 3 .

The case of rank 0 , any $d$, is trivial since here $\mathcal{A}=\emptyset$ and $\Phi$ has only one facet $C=M$; so, $\mathbf{S}^{(d)}$ is given by the unique point $e(C, \ldots, C)$, and $\Gamma^{(d)}=\emptyset$ verifies the thesis.

We assume the theorem holds when the arrangement has rank lower than $r k(\mathcal{A})$ and arbitrary degree, or when the arrangement has the same rank as $\mathcal{A}$ but the degree $d^{\prime}$ is lower than $d$.

To prove 1), we partition $\Gamma_{(d)}$ into subsets, each of these being a discrete field; this will suffice to prove that $\Gamma_{(d)}$ is a field.

Consider first all cells in $\mathbf{S}^{(d)}$ with $\operatorname{codim}\left(F_{1}\right)=0$, that is of the kind

$$
e\left(C, C, F_{2}, \ldots, F_{d}\right) \text {. }
$$

This set bijectively corresponds to $\mathbf{S}^{(d-1)}$ by $j_{d-1}$.

By induction, $\Gamma_{(d-1)}$ is a discrete field, so $\lambda_{*}\left(\Gamma_{(d-1)}\right) \subset \Gamma_{(d)}$ is also a discrete field (see 4.7). The cells of $j_{d-1}\left(\mathbf{S}^{(d-1)}\right) \subset \mathbf{S}^{(d)}$ (of the shape (9)) which are not contained in $\lambda_{*}\left(\Gamma_{(d-1)}\right)$ are exactly given by

$$
j_{d-1}\left(\operatorname{Sing}\left(\mathbf{S}^{(d-1)}\right)\right)=\left\{j_{d-1}(e(\mathcal{F})): \quad e(\mathcal{F}) \text { critical cell of } \mathbf{S}^{(d-1)}\right\} .
$$

By induction, the cells (10) will be of the following form:

$$
e\left(C, C, F^{h}, \ldots, F^{h}\right)
$$

$$
\text { with } e\left(C, F^{h}\right) \text { critical for }\left(\Gamma_{(1)}, \triangleleft\right) \text { if } d \text { is even; }
$$

$$
e\left(o p_{F^{h}}(C), o p_{F^{h}}(C), F^{h}, \ldots, F^{h}\right)
$$

with $e\left(C, F^{h}\right)$ critical for $\left(\Gamma_{(1)}, \triangleleft\right)$ if $d$ is odd

(of course, $d$ even implies $d-1$ odd and conversely).

Notice that the unique 0 -cell in $(10)$ is $e\left(C_{0}, \ldots, C_{0}\right)$, where $C_{0}$ is the unique chamber containing the origin $V_{0}$ of the polar system. All the other $0-$ cells in $\mathbf{S}^{(d)}$ (all having analog shape $\left.e(C, \ldots, C), C \neq C_{0}\right)$ belong to some pair in the image of $\lambda_{*}$.

It will be useful to give an equivalent condition for a cell $e\left(C, F^{h}\right) \in \mathbf{S}$ to be critical, which come easily from point (4) of theorem 2 :

$$
F^{h} \cap V^{h} \neq \emptyset \text { and } p r_{\left|F^{h}\right|}(C) \cap V^{h-1} \neq \emptyset \text { and bounded. }
$$

Now let $L \in \mathcal{L}(\mathcal{A})$ be a codim- $k$ subspace, $k>0$. Consider all cells in $\mathbf{S}^{(d)}$ of the form

$$
\left\{e\left(C, F^{k}, F_{2}, \ldots, F_{d}\right) \mid F^{k} \subset L\right\} .
$$

By induction $\Gamma_{(d-1)}^{L}(4.6)$ is a discrete vector field. By the definition of $\Gamma_{(d)}$, one has that $\lambda_{*}\left(\Gamma_{(d-1)}^{L}\right)$ is contained into $\Gamma_{(d)}$ and by 4.7 it is a discrete field. 
The remaining cells of $\mathbf{S}^{(d)}$ of shape (14) which are not in $\lambda_{*}\left(\Gamma_{(d-1)}^{L}\right)$ (i.e. the cells in $\left.\lambda\left(\operatorname{Sing}\left(\mathbf{S}^{(d-1)}\left(\mathcal{A}^{L}\right)\right)\right)\right)$ have the form

$$
e\left(C, F^{k}, F^{h}, \ldots, F^{h}\right)
$$

with $e\left(F^{k}, F^{h}\right)$ critical cell for $\left(\Gamma_{(1)}^{L}, \triangleleft^{L}\right)$ for even $d$;

$$
e\left(C, o p_{F^{h}}\left(F^{k}\right), F^{h}, \ldots, F^{h}\right)
$$

with $e\left(F^{k}, F^{h}\right)$ critical cell for $\left(\Gamma_{(1)}^{L}, \triangleleft^{L}\right)$ for odd $d$.

In particular for $h=k$ they remain all cells $e\left(C, F_{0}^{k}, \ldots, F_{0}^{k}\right)$ for all $C \prec F_{0}^{k}$, where $F_{0}^{k}$ is the unique facet in $L$ such that $F_{0}^{k} \cap V^{k} \neq \emptyset$.

When $k<h$ condition (13) for critical cells translates as:

$$
\begin{gathered}
V^{h} \cap L \cap F^{h}=V^{h} \cap F^{h} \neq \emptyset \\
\text { and } \quad p r_{\left|F^{h}\right|}\left(F^{k}\right) \cap V^{h-1} \cap L=p r_{\left|F^{h}\right|}\left(F^{k}\right) \cap V^{h-1} \neq \emptyset \quad \text { and bounded. }
\end{gathered}
$$

Let $L^{\prime}, L^{\prime \prime} \in \mathcal{L}(\mathcal{A})$. Remark that $\Gamma_{(d-1)}^{L^{\prime}}$ and $\Gamma_{(d-1)}^{L^{\prime \prime}}$ have no common cell if $L^{\prime} \neq L^{\prime \prime}$ (by definition, the first facet defining a cell has support respectively $L^{\prime}, L^{\prime \prime}$ ). When $L$ is the whole space $M$, clearly $\Gamma_{(d-1)}=\Gamma_{(d-1)}^{L}$. If $L=L_{0}=\cap\left\{H_{i}\right\}$ is the center of the arrangement, $\Gamma_{(d-1)}^{L}=\emptyset$ and the unique critical cell is $e_{0}:=e(L, \ldots, L)$. In this case $\lambda\left(e_{0}\right)=\{e(C, L, \ldots, L) \mid C$ any chamber $\}$.

Summarizing the previous discussion, we have by induction that

$$
\Gamma_{(d)}^{\prime}=\bigcup_{L \in \mathcal{L}(\mathcal{A})} \lambda_{*}\left(\Gamma_{(d-1)}^{L}\right) \subset \Gamma_{(d)}
$$

is a discrete field; the set $\mathcal{E}$ of cells of $\mathbf{S}^{(d)}$ which do not belong to $\Gamma_{(d)}^{\prime}$ are given by

$$
e\left(C, F^{k}, F^{h}, \ldots, F^{h}\right)
$$

with $F^{h} \cap V^{h} \neq \emptyset, \operatorname{pr}_{\left|F^{h}\right|}\left(F^{k}\right) \cap V^{h-1} \neq \emptyset$ and bounded, even $d$,

$$
e\left(C, o p_{F^{h}}\left(F^{k}\right), F^{h}, \ldots, F^{h}\right)
$$

with $F^{h} \cap V^{h} \neq \emptyset, p r_{\left|F^{h}\right|}\left(F^{k}\right) \cap V^{h-1} \neq \emptyset$ and bounded, odd $d$,

when $k<h$; by

$$
e\left(C, F^{h}, \ldots, F^{h}\right)
$$

$$
\text { with } F^{h} \cap V^{h} \neq \emptyset
$$

when $h=k$.

The proof of parts (1) and (3) of theorem 3 will follow from the following lemma. 
Lemma 4.8. Let $k<h$. For each cell $e\left(C, F^{k}, F^{h}, \ldots, F^{h}\right) \in \mathcal{E}$, there exists either

$$
F^{k+1} \text { such that } F^{k} \prec F^{k+1} \prec F^{h} \text { (if } k+1=h \text { then } F^{k+1}=F^{h} \text { ) }
$$

or

$$
F^{k-1} \text { such that } C \prec F^{k-1} \prec F^{k} \text { (if } k=1 \text { then } F^{k-1}=C \text { ) }
$$

such that $e\left(C, F^{k+1}, F^{h}, \ldots, F^{h}\right) \in \mathcal{E}\left(\right.$ resp. $\left.e\left(C, F^{k-1}, F^{h}, \ldots, F^{h}\right) \in \mathcal{E}\right)$ and the pair $\left(e\left(C, F^{k}\right), e\left(C, F^{k+1}\right)\right)$ (resp. $\left.\left(e\left(C, F^{k-1}\right), e\left(C, F^{k}\right)\right)\right)$ belongs to the field $\Gamma_{L,(1)}$ which is defined by using the ordering

$$
\triangleleft_{L}^{o p} \quad, \text { for even } d ;
$$

or

$$
\triangleleft_{L} \quad \text {, for odd } d \text {. }
$$

$\left(L:=\left|F^{h}\right|\right)$.

Case $k=h$. For each cell $e\left(C, F^{k}, \ldots, F^{k}\right) \in \mathcal{E}$, there exists $F^{k-1}$ verifying (22) and such that $e\left(C, F^{k-1}, F^{k}, \ldots, F^{k}\right)$ belongs to $\mathcal{E}$ and the pair $\left(e\left(C, F^{k-1}\right), e\left(C, F^{k}\right)\right)$ belongs to the field $\left(\Gamma_{L,(1)}, \triangleleft_{L}^{o p}\right)$ for $d$ even, respectively to $\left(\Gamma_{L,(1)}, \triangleleft_{L}\right)$ for $d$ odd, $\left(L=\left|F^{k}\right|\right)$ iff $e\left(C, F^{k}\right)$ is non critical for $\left(\Gamma_{L,(1)}, \triangleleft_{L}^{o p}\right)$ for $d$ even, respectively for $\left(\Gamma_{L,(1)}, \triangleleft_{L}\right)$ for $d$ odd.

Proof of lemma 4.8. We show first that $e\left(C, F^{k}\right)$ is not critical for $\Gamma_{L,(1)}$ (with the suitable ordering defined above).

Assume $d$ even. Condition (18), giving $p r_{\left|F^{h}\right|}\left(F^{k}\right) \cap V^{h-1} \neq \emptyset$ and bounded, is equivalent to

$$
\operatorname{pr}_{\mid F^{h \mid}}\left(o p_{F^{h}}\left(F^{k}\right)\right) \cap V^{h-1}=\emptyset .
$$

If $e\left(C, F^{k}\right)$ were critical for $\left(\Gamma_{L,(1)}, \triangleleft_{L}^{o p}\right)$ then $p r_{\left|F^{h}\right|}\left(o p_{F^{h}}\left(F^{k}\right)\right) \cap V^{k} \neq \emptyset$. Since $V^{k} \subset V^{h-1}$ this is impossible.

If $d$ is odd, condition (19), giving $\operatorname{pr}_{\left|F^{h}\right|}\left(o p_{F^{h}}\left(F^{k}\right)\right) \cap V^{h-1} \neq \emptyset$ and bounded, is equivalent to $p r_{\left|F^{h}\right|}\left(F^{k}\right) \cap V^{h-1}=\emptyset$. If $e\left(C, F^{k}\right)$ were critical for $\left(\Gamma_{L,(1)}, \triangleleft_{L}\right)$ then $p r_{\left|F^{h}\right|}\left(F^{k}\right) \cap V^{k} \neq \emptyset$, and this also is impossible.

Assume that there exists $F^{k+1}$ as in (21) such that the pair

$$
\left(e\left(C, F^{k}\right), e\left(C, F^{k+1}\right)\right) \in \Gamma_{L,(1)} .
$$

We have to show that $e\left(C, F^{k+1}, F^{h}, \ldots, F^{h}\right) \in \mathcal{E}$.

In case $k+1<h$, if condition (18) holds for $F^{k}$ ( $d$ even) then it clearly holds for all facets $F^{k+1}$ in the boundary of $F^{k}$ and such that $F^{k+1} \prec F^{h}$, and analog for condition (19).

When $k+1=h$, then by (20) all cells $e\left(C, F^{h}, \ldots, F^{h}\right)$ with $F^{h} \cap V^{h} \neq \emptyset$ belong to $\mathcal{E}$.

Now let us suppose there exists $F^{k-1}$ as in (22) such that

$$
\left(e\left(C, F^{k-1}\right), e\left(C, F^{k}\right)\right) \in \Gamma_{L,(1)}
$$

and we have to show that $e\left(C, F^{k-1}, F^{h}, \ldots, F^{h}\right) \in \mathcal{E}$. 
Let $d$ be even. By the definition of $\left(\Gamma_{L,(1)}, \triangleleft_{L}^{o p}\right)$ (section 3) one must have $F^{k} \triangleleft_{L}^{o p}$ $F^{k-1}$, that is $o p_{F^{h}}\left(F^{k}\right) \triangleleft_{L} o p_{F^{h}}\left(F^{k-1}\right)$. Since for $F^{k}$ condition (23) holds, we must have (by definition of polar ordering) also

$$
\operatorname{pr}_{\left|F^{h}\right|}\left(o p_{F^{h}}\left(F^{k-1}\right)\right) \cap V^{h-1}=\emptyset
$$

which is equivalent (as said above) to (18), so it gives the thesis in this case.

The case $d$ odd is proved in the same way, so as the last assertion of the theorem for case $k=h$.

From lemma 4.8 it follows both parts 1) and 3) of theorem 3. Indeed, the pairs of cells of $\Gamma_{(d)}$ coming from lemma 4.8 clearly do not have common cells and, together with the previous field $\Gamma_{(d)}^{\prime}$, exhaust all $\Gamma_{(d)}$. This proves part 1). Part 3) follows from the last part of lemma 4.8 .

We now come to part 2) of the theorem. We use the characterizing property for gradient fields: every closed $\Gamma_{(d)}$-path is trivial ([7]; see the comments soon after theorem 2).

Take a $\Gamma_{(d)}$-path in $\mathbf{S}^{(d)}$ :

$$
e\left(C_{1}, \mathcal{F}_{1}^{k}\right), e\left(C_{1}, \mathcal{F}_{1}^{k+1}\right), \ldots, e\left(C_{m}, \mathcal{F}_{m}^{k}\right), e\left(C_{m}, \mathcal{F}_{m}^{k+1}\right), e\left(C_{m+1}, \mathcal{F}_{m+1}^{k}\right)
$$

where by $\mathcal{F}_{i}^{k}$ we indicate the flag $\left(F_{i, 1}^{j_{i, 1}}, \ldots, F_{i, d}^{j_{i, d}}\right)$, whose codimension is $k=\sum_{l=1}^{d} j_{i, l}$, and by $\mathcal{F}_{i}^{k+1}$ we indicate the flag $\left(F_{i, 1}^{j_{i, 1}^{\prime}}, \ldots, F_{i, d}^{j_{i, d}^{\prime}}\right)$, whose codimension is $k+1=$ $\sum_{l=1}^{d} j_{i, l}^{\prime}(i=1, \ldots, m)$. Here the pair $\left(e\left(C_{i}, \mathcal{F}_{i}^{k}\right), e\left(C_{i}, \mathcal{F}_{i}^{k+1}\right)\right)$ is an element of $\Gamma_{(d)}$, and $e\left(C_{i}, \mathcal{F}_{i}^{k}\right)$ is in the boundary of $e\left(C_{i-1}, \mathcal{F}_{i-1}^{k+1}\right)$.

We have to prove that if the path (24) is closed (i.e. if $e\left(C_{m+1}, \mathcal{F}_{m+1}^{k}\right)$ equals $\left.e\left(C_{1}, \mathcal{F}_{1}^{k}\right)\right)$, then such path is trivial, i.e. $\mathcal{F}_{i}^{k}=\mathcal{F}_{i+1}^{k}, \mathcal{F}_{i}^{k+1}=\mathcal{F}_{i+1}^{k+1}$, and $C_{i}=C_{i+1}$ $(i=1, \ldots, m-1)$.

Let $\varangle$ be a polar ordering over the face poset $\Phi\left(\mathcal{A}^{\prime}\right)$ of an arrangement $\mathcal{A}^{\prime}$. Introduce the ordering $\triangleleft_{\text {lex }}$ in $\Phi^{(d)}\left(\mathcal{A}^{\prime}\right)$

$$
\left(F_{0}, F_{1}, \ldots, F_{d}\right) \triangleleft_{l e x}\left(F_{0}^{\prime}, F_{1}^{\prime}, \ldots, F_{d}^{\prime}\right)
$$

$\left(\operatorname{codim}\left(F_{0}\right)=\operatorname{codim}\left(F_{0}^{\prime}\right)=0\right)$ iff, being $\underline{k}$ the last position where $F_{k} \neq F_{k}^{\prime}$, one has

$$
F_{\underline{k}} \boldsymbol{\Psi}_{\underline{k}} F_{\underline{k}}^{\prime},
$$

where $\boldsymbol{\iota}_{\underline{k}}$ equals either $\boldsymbol{\triangleleft}_{\mid F_{\underline{k}}+1}$ or $\boldsymbol{\triangleleft}_{\left|F_{\underline{k}+1}\right|}^{o p}$ according to the parity of $d-\underline{k}$.

We will prove the following claim:

Claim 3. Given a triple of consecutive cells in (24) of the form:

$$
e\left(C_{i}, \mathcal{F}_{i}^{k+1}\right), e\left(C_{i+1}, \mathcal{F}_{i+1}^{k}\right), e\left(C_{i+1}, \mathcal{F}_{i+1}^{k+1}\right) .
$$

we have that $\mathcal{F}_{i+1}^{k+1} \unlhd_{\text {lex }} \mathcal{F}_{i}^{k+1}$.

We prove this claim inductively on $d$. The base of the induction is the case $d=1$, i.e. claim 1 of section 3 .

We now suppose the statement true for $d-1$ and prove it for $d$.

By splitting a flag $\mathcal{F}=\left(\mathcal{G}, F_{d}\right)$ into a $(d-1)$-flag $\mathcal{G}$ and the last facet $F_{d}$, the triple (25) will become: 


$$
e\left(C_{i}, \mathcal{G}_{i}^{h_{i}^{\prime}}, F_{i, d}^{l_{i}^{\prime}}\right), e\left(C_{i+1}, \mathcal{G}_{i+1}^{h_{i+1}}, F_{i+1, d}^{l_{i+1}}\right), e\left(C_{i+1}, \mathcal{G}_{i+1}^{h_{i+1}^{\prime}}, F_{i+1, d}^{l_{i+1}^{\prime}}\right) .
$$

Now we have to distinguish several cases.

(1) If $l_{i}^{\prime}=l_{i+1}=l_{i+1}^{\prime}=l$, then $F_{i+1, d}^{l} \prec F_{i, d}^{l}$ and so $F_{i, d}^{l}=F_{i+1, d}^{l}$ by a dimension argument. We have also that $h_{i}^{\prime}-1=h_{i+1}=h_{i+1}^{\prime}-1=h$, so we can rewrite the triple as:

$$
e\left(C_{i}, \mathcal{G}_{i}^{h+1}, F^{l}\right), e\left(C_{i+1}, \mathcal{G}_{i+1}^{h}, F^{l}\right), e\left(C_{i+1}, \mathcal{G}_{i+1}^{h+1}, F^{l}\right) .
$$

By inductive hypothesis we have that $\mathcal{G}_{i+1}^{h+1}\left(\triangleleft_{F^{l}}^{o p}\right)_{l e x} \mathcal{G}_{i}^{h+1}$, and so also $\mathcal{F}_{i+1}^{k+1} \triangleleft_{\text {lex }} \mathcal{F}_{i}^{k+1}$.

(2) If $l_{i}^{\prime}-1=l_{i+1}=l_{i+1}^{\prime}-1=l$, so $h_{i}^{\prime}=h_{i+1}=h_{i+1}^{\prime}=h$ and we can write the triple as:

$$
e\left(C_{i}, \mathcal{G}_{i}^{h}, F_{i, d}^{l+1}\right), e\left(C_{i+1}, \mathcal{G}_{i+1}^{h}, F_{i+1, d}^{l}\right), e\left(C_{i+1}, \mathcal{G}_{i+1}^{h}, F_{i+1, d}^{l+1}\right) .
$$

This case follows from claim 1 applied to the triple

$$
e\left(F_{i, d-1}, F_{i, d}^{l+1}\right), e\left(F_{i+1, d-1}, F_{i+1, d}^{l}\right), e\left(F_{i+1, d-1}, F_{i+1, d}^{l+1}\right)
$$

in the arrangement $\mathcal{A}^{L}, L=\left|F_{i+1, d-1}\right|$.

(3) If $l_{i}^{\prime}-1=l_{i+1}=l_{i+1}^{\prime}=l$, so $h_{i}^{\prime}=h_{i+1}=h_{i+1}^{\prime}-1=h$ and we can write the triple as:

$$
e\left(C_{i}, \mathcal{G}_{i}^{h}, F_{i, d}^{l+1}\right), e\left(C_{i+1}, \mathcal{G}_{i+1}^{h}, F_{i+1, d}^{l}\right), e\left(C_{i+1}, \mathcal{G}_{i+1}^{h+1}, F_{i+1, d}^{l}\right) .
$$

Here $\left(e\left(C_{i+1}, \mathcal{G}_{i+1}^{h}, F_{i+1, d}^{l}\right), e\left(C_{i+1}, \mathcal{G}_{i+1}^{h+1}, F_{i+1, d}^{l}\right)\right) \in \Gamma_{(d)}$, so, by condition (5), there exists an index $j \geq 1$ so that $e\left(F_{i+1, d-j}, F_{i+1, d-j+1}, \ldots, F_{i+1, d}^{l}\right)$ is critical for $\Gamma_{(j)}^{L}$, where $L=\left|F_{i+1, d-j}\right|$; we have in particular $F_{i+1, d}^{l} \cap V^{l} \neq \emptyset$. The definition of polar ordering gives $F_{i+1, d}^{l} \triangleleft F_{i, d}^{l+1}$, so $\mathcal{F}_{i+1}^{k+1} \triangleleft_{\text {lex }} \mathcal{F}_{i}^{k+1}$ as required.

(4) If $l_{i}^{\prime}=l_{i+1}=l_{i+1}^{\prime}-1=l$, so $F_{i, d}^{l}=F_{i+1, d}^{l}$ as above, and $h_{i}^{\prime}-1=h_{i+1}=$ $h_{i+1}^{\prime}=h$, so we can write the triple as:

$$
e\left(C_{i}, \mathcal{G}_{i}^{h+1}, F^{l}\right), e\left(C_{i+1}, \mathcal{G}_{i+1}^{h}, F^{l}\right), e\left(C_{i+1}, \mathcal{G}_{i+1}^{h}, F^{l+1}\right) .
$$

Since $\left(e\left(C_{i+1}, \mathcal{G}_{i+1}^{h}, F^{l}\right), e\left(C_{i+1}, \mathcal{G}_{i+1}^{h}, F^{l+1}\right)\right) \in \Gamma_{(d)}$ then condition (6) gives $F^{l+1} \triangleleft F^{l}$ and $\mathcal{F}_{i+1}^{k+1} \triangleleft_{l e x} \mathcal{F}_{i}^{k+1}$ as required.

This proves the claim. If the path (24) is closed, it follows that all the flags of codimension $k+1$ equal a fixed flag $\mathcal{F}^{k+1}$.

We can rewrite (24) as follows:

$$
e\left(C_{1}, \mathcal{F}_{1}^{k}\right), e\left(C_{1}, \mathcal{F}^{k+1}\right), \ldots, e\left(C_{m}, \mathcal{F}_{m}^{k}\right), e\left(C_{m}, \mathcal{F}^{k+1}\right), e\left(C_{1}, \mathcal{F}_{1}^{k}\right) .
$$

So $\mathcal{F}_{i}^{k} \prec \mathcal{F}^{k+1}, i=1, \ldots, m$.

We will now prove another claim: 
Claim 4. Given a quadruple of consecutive cells in (26) of the form:

$$
e\left(C_{i}, \mathcal{F}_{i}^{k}\right), e\left(C_{i}, \mathcal{F}^{k+1}\right), e\left(C_{i+1}, \mathcal{F}_{i+1}^{k}\right), e\left(C_{i+1}, \mathcal{F}^{k+1}\right) .
$$

we have $\mathcal{F}_{i}^{k} \unlhd_{\text {lex }} \mathcal{F}_{i+1}^{k}$.

We prove this claim inductively on $d$. The base of the induction is the case $d=1$, i.e. claim 2 of section 3 .

We now suppose the statement true for $d-1$ and prove it for $d$.

As before, we can rewrite the quadruple as:

$$
e\left(C_{i}, \mathcal{G}_{i}^{h_{i}}, F_{i, d}^{l_{i}}\right), e\left(C_{i}, \mathcal{G}^{h+1}, F^{l+1}\right), e\left(C_{i+1}, \mathcal{G}_{i+1}^{h_{i+1}}, F_{i+1, d}^{l_{i+1}}\right), e\left(C_{i+1}, \mathcal{G}^{h+1}, F^{l+1}\right) .
$$

Let us distinguish several cases:

(1) if $l_{i}=l_{i+1}=l+1$ then, by definition of $\Gamma_{(d)}$, it follows $F_{i, d}^{l+1}=F^{l+1}=F_{i+1, d}^{l+1}$ and $h_{i}=h_{i+1}=h$, so the quadruple becomes:

$$
e\left(C_{i}, \mathcal{G}_{i}^{h}, F^{l+1}\right), e\left(C_{i}, \mathcal{G}^{h+1}, F^{l+1}\right), e\left(C_{i+1}, \mathcal{G}_{i+1}^{h}, F^{l+1}\right), e\left(C_{i+1}, \mathcal{G}^{h+1}, F^{l+1}\right)
$$

By inductive hypothesis we have $\mathcal{G}_{i}^{h}\left(\triangleleft_{F^{l+1}}^{o p}\right)_{l e x} \mathcal{G}_{i+1}^{h}$, and consequently also $\mathcal{F}_{i}^{k} \triangleleft_{\text {lex }} \mathcal{F}_{i+1}^{k}$.

(2) If $l_{i}=l_{i+1}=l$ then $h_{i}=h_{i+1}=h+1$ and the quadruple become:

$$
\begin{gathered}
e\left(C_{i}, \mathcal{G}^{h+1}, F_{i, d}^{l}\right), e\left(C_{i}, \mathcal{G}^{h+1}, F^{l+1}\right), e\left(C_{i+1}, \mathcal{G}^{h+1}, F_{i+1, d}^{l}\right), \\
, e\left(C_{i+1}, \mathcal{G}^{h+1}, F^{l+1}\right)
\end{gathered}
$$

(the fact that all the $\mathcal{G}$ 's coincide here follows directly from the definition of $\Gamma_{(d)}$ applied to both pairs of the field in (27)).

From claim 2, applied to the quadruple

$$
e\left(G, F_{i, d}^{l}\right), e\left(G, F^{l+1}\right), e\left(G, F_{i+1, d}^{l}\right), e\left(G, F^{l+1}\right),
$$

where $G$ is the last facet of $\mathcal{G}$, it follows $F_{i, d}^{l} \unlhd F_{i+1, d}^{l}$, which concludes this case.

(3) if $l_{i}=l_{i+1}+1=l+1$ then $F_{i, d}^{l+1}=F^{l+1}$, moreover $h_{i}+1=h_{i+1}=h+1$. So the quadruple becomes:

$$
e\left(C_{i}, \mathcal{G}_{i}^{h}, F^{l+1}\right), e\left(C_{i}, \mathcal{G}^{h+1}, F^{l+1}\right), e\left(C_{i+1}, \mathcal{G}^{h+1}, F_{i+1}^{l}\right), e\left(C_{i+1}, \mathcal{G}^{h+1}, F^{l+1}\right) .
$$

By condition (6) we have $F^{l+1} \triangleleft F_{i+1}^{l}$, so $\mathcal{F}_{i}^{k} \triangleleft_{l e x} \mathcal{F}_{i+1}^{k}$ as required.

(4) if $l_{i}+1=l_{i+1}=l+1$ then $F_{i+1, d}^{l+1}=F^{l+1}$, moreover $h_{i}=h_{i+1}+1=h+1$. So the quadruple becomes:

$$
e\left(C_{i}, \mathcal{G}^{h+1}, F_{i, d}^{l}\right), e\left(C_{i}, \mathcal{G}^{h+1}, F^{l+1}\right), e\left(C_{i+1}, \mathcal{G}_{i+1}^{h}, F^{l+1}\right), e\left(C_{i+1}, \mathcal{G}^{h+1}, F^{l+1}\right) .
$$

It follows from condition (5) that there exists an index $j$ such that

$$
e\left(F_{i+1, j}^{p}, F_{i+1, j+1}=F^{l+1}, \ldots, F^{l+1}\right)
$$

is critical for $\Gamma_{(d-j)}^{L}$, with $L=\left|F_{i+1, j}^{p}\right|$.

By construction $\mathcal{G}_{i+1}^{h}, \mathcal{G}_{h+1}$ differ only in the position $j$ : 


$$
\begin{aligned}
\mathcal{G}_{i+1}^{h} & =\left(F_{i+1,1}, \ldots, F_{i+1, j-1}, F_{i+1, j}^{p}, F_{i+1, j+1}, \ldots, F_{i+1, d-1}\right), \\
\mathcal{G}^{h+1} & =\left(F_{i+1,1}, \ldots, F_{i+1, j-1}, F_{i+1, j}^{p+1}, F_{i+1, j+1}, \ldots, F_{i+1, d-1}\right) .
\end{aligned}
$$

Since $F_{i+1, k}=F^{l+1}$ for $k>j$, we deduce $j=d-1$, so:

$$
\begin{aligned}
\mathcal{G}_{i+1}^{h} & =\left(F_{i+1,1}, \ldots, F_{i+1, d-2}, F_{i+1, d-1}^{p}\right) \\
\mathcal{G}^{h+1} & =\left(F_{i+1,1}, \ldots, F_{i+1, d-2}, F_{i+1, d-1}^{p+1}\right) .
\end{aligned}
$$

So $e\left(F_{i+1, d-1}^{p}, F^{l+1}\right)$ is critical in $\left(\Gamma_{(1)}^{L}, \triangleleft\right), L=\left|F_{i+1, d-1}^{p}\right|$. It follows:

$$
F^{l+1}=\max _{\triangleleft}\left\{F \mid F_{i+1, d-1}^{p} \prec F \prec F^{l+1}\right\} .
$$

We have also $F_{i+1, d-1}^{p} \prec F_{i+1, d-1}^{p+1} \prec F_{i, d}^{l} \prec F^{l+1}$, which gives $F_{i, d}^{l} \triangleleft F^{l+1}$. This is not possible since condition (6) gives $F^{l+1} \triangleleft F_{i, d}^{l}$.

Then all $\mathcal{F}_{i}^{k}$ coincide.

It remains to see that all the chambers $C_{i}$ 's coincide. We will show that $C_{i}=C_{i+1}$ for $i=1 \ldots m-1$.

In (26) we can take a triple of consecutive cells of the form:

$$
e\left(C_{i}, \mathcal{F}^{k}\right), e\left(C_{i}, \mathcal{F}^{k+1}\right), e\left(C_{i+1}, \mathcal{F}^{k}\right)
$$

where the pair $\left(e\left(C_{i}, \mathcal{F}^{k}\right), e\left(C_{i}, \mathcal{F}^{k+1}\right)\right)$ is in $\Gamma_{(d)}$ and $e\left(C_{i+1}, \mathcal{F}^{k}\right)$ is in the boundary of $e\left(C_{i}, \mathcal{F}^{k+1}\right)$. If we write $\mathcal{F}^{k}=\left(F_{1}, \mathcal{F}^{\prime}\right)$ then this triple become:

$$
e\left(C_{i}, F_{1}, \mathcal{F}^{\prime}\right), e\left(C_{i}, \mathcal{F}^{k+1}\right), e\left(C_{i+1}, F_{1}, \mathcal{F}^{\prime}\right) .
$$

By the boundary condition (lemma 2.2) we have $p r_{\left|F_{1}\right|}\left(C_{i+1}\right) \prec p r_{\left|F_{1}\right|}\left(C_{i}\right)$. By a dimension argument, it follows $p r_{\left|F_{1}\right|}\left(C_{i+1}\right)=p r_{\left|F_{1}\right|}\left(C_{i}\right)$. Since $C_{i} \prec F_{1}$ and $C_{i+1} \prec$ $F_{1}$, it follows $C_{i}=C_{i+1}$.

This completes the proof.

\section{References}

[1] A.Bjorner and G.Ziegler, Combinatorial stratifications of complex arrangements, Jour. Amer. Math. Soc. 5 (1992) 105-149.

[2] C. D. Concini and M. Salvetti, Cohomology of Coxeter groups and Artin groups, Math. Res. Lett. 7 (2000), no. 2-3, 213-232.

[3] E. Delucchi, Shelling-Type Orderings of Regular CW-Complexes and Acyclic Martchings of the Salvetti Complex, Int. Math. Res. Notices 6 (2008)

[4] E. Delucchi and S. Settepanella, Combinatorial polar ordering and recursively orderable arrangements, Adv. in Appl. Math. 44 (2009), no. 1, 124-144.

[5] A. Dimca and S. Papadima, Hypersurface complements, Milnor fibers and higher homotopy groups of arrangements, Ann. of Math. 158 (2003), no. 2, 473-507.

[6] R. Forman, Morse Theory for Cell Complexes, Adv. in Math. 134 (1998), no. 1, 90-145.

[7] — A User's guide to discrete Morse Theory, Sem. Lotharingien de Combinatoire 48 (2002)

[8] G.Gaiffi and M.Salvetti, The Morse complex of a line arrangement, Jour. of Algebra 321 (2009) 316-337.

[9] M. Goreski and R. MacPherson, Stratified Morse Theory, Vol. 14, Ergebnisse der Mathematik und ihrer Grenzgebiete Springer-Verlag, Berlin Heidelberg New York (1988).

[10] D. Kozlov, Combinatorial Algebraic Topology, Vol. 31 of Algorithms and Computation in Mathematics, Springer (2008). 
[11] M.Salvetti, Topology of the complement of real hyperplanes in $\mathbb{C}^{n}$, Inv. Math. 88 (1987), no. 3, 603-618.

[12] M.Salvetti and S. Settepanella, Combinatorial Morse theory and Minimality of Hyperplane Arrangements, Geom. and Top. 11 (2007) 1733-1765.

[13] P. Orlik and M. Terao, Arrangements of hyperplanes, Vol. 300, Springer-Verlag (1992).

[14] R. Randell, Morse theory, Milnor fibers and minimality of a complex hyperplane arrangement, Proc. Amer. Math. Soc. 130 (2002), no. 9, 2737-2743.

[15] T.Zaslavsky, Facing up to arrangements: face count formulas for partitions of space by hyperplanes, Memoirs Amer. Math. Soc. 1 (1975), no. 154, 1-102.

[16] M. Yoshinaga, Hyperplane arrangements and Lefschetz's hyperplane section theorem, Kodai Math. J. (2007), no. 2, 157-194.

Dipartimento di Matematica "L. Tonelli", University of Pisa, Largo B. Pontecorvo 5, 56127 PisA, ITALY

E-mail address: mori@mail.dm.unipi.it

Dipartimento di Matematica "L. Tonelli", University of Pisa, Largo B. Pontecorvo 5, 56127, Pisa, Italy (Partially Supported By M.U.R.S.T. 40\%)

E-mail address: salvetti@dm.unipi.it 